\title{
VLBI follow-up observations for the large X-ray flares of Mrk 421
}

\author{
Kotaro Niinuma* \\ Yamaguchi University, Yoshida 1677-1, Yamaguchi, Yamaguchi 753-8512, Japan \\ E-mail: niinumadyamaguchi-u.ac.jp
}

Motoki Kino, Akihiro Doi, Naoki Isobe

ISAS / JAXA, Yoshinodai, Chuo, Sagamihara, Kanagawa 252-5210, Japan

\section{Shoko Koyama}

The University of Tokyo, Hongo, Bunkyo-ku, Tokyo 113-8654, Japan

In February 2010 and September 2011, the large X-ray flares of Mrk 421 were reported by the Astronomer's Telegram. Mrk 421 is one of the best sources for studying the most compact regions in blazars, because of its proximity. In order to examine the behavior inside the sub-pc region by densely VLBI monitor soon after the large flare, we carried out quick and multi-epoch follow-up observations for these flares using JVN (the Japanese VLBI network) array at an interval of two weeks to three weeks. As results of our VLBI observations for these large X-ray flares, we present the possibility of a structural change that occurred within $\sim 1.2$ mas from the core after the large high-energy flares.

11th European VLBI Network Symposium \& Users Meeting,

October 9-12, 2012

Bordeaux, France

* Speaker. 


\section{High-energy flares of Mrk 421}

Mrk 421 is one of the nearest and the first-discovered TeV blazar $(z=0.031, \mathrm{D}=130 \mathrm{Mpc}$, $1 \mathrm{mas}=0.61 \mathrm{pc})$. Therefore this source is one of the best target to study the 'blazar zone' using VLBI. Also the large high-energy flares of Mrk 421 have been frequently reported. Since the first discovery of VHE $\gamma$-ray emission from Mrk 421 was reported by Punch et al. [四], the large X-ray or VHE $\gamma$-ray flares of Mrk 421 have occasionally been reported. Gaidos et al. [D] succeeded in detecting the VHE $\gamma$-ray flares from Mrk 421 within a few years after the discovery of VHE emission. An extremely high state of Mrk 421 at VHE band, which lasted from 2001 January to March, was reported by Krennrich et al. [B]]. Recently, multi-band observations have also been performed for such large flares of Mrk 421 ([䧃-团]). These flares are summarized in Table 1 of Niinuma et al. [四].

Recently, several large X-ray flares have been detected by MAXI (Monitor of All-sky X-ray Image: [ [8]]), and/or Swift/BAT ([Q]]). These X-ray flares are clearly seen in the X-ray light curves of MAXI and Swift/BAT between 2009 August and 2012 June which are shown in Figure $\mathbb{m}$. In order to study the behavior of the blazar core right after the large flare, we conducted a quick and dense monitor campaign of observations of Mrk 421 using JVN for the 2010 February flare alerted by Isobe et al. [ए]], and for the 2011 September flare alerted by Hiroi et al. [ए]] shown in Figure 凹. Additionally, the 2010 February flare is the largest one among those ever reported ([ए]]).

\section{VLBI observations and data analysis}

We show the two observing sessions (A, B in Figure $\mathrm{W}$ ) at which we conducted VLBI observations of the large X-ray flares of Mrk 421. In session A, we observed during five epochs using JVN ([[3] ) at an interval of $\sim 20$ days from 19 days after the largest X-ray flare occurred. In session $\mathrm{B}$, we carried out a total of thirteen astrometric observations using VERA (VLBI Exploration of Radio Astrometry [4]], which is also included in JVN) at an interval of $10-20$ days from 9 days after the X-ray flare occurred.

For the correlated data, we performed the standard VLBI calibration and fringe-fitting procedure using the NRAO Astronomical Image Processing System (AIPS) software package. The flux calibration done by the AIPS task APCAL typically achieved an accuracy of $10 \%$, for VERA at $22 \mathrm{GHz}$ ([[5]]). The calibrated visibilities were then exported to carry out an imaging procedure using the Caltech Difmap package. After the time averaging of visibility data in 30-s bins, we carried out the iterations of CLEAN, and self-calibration procedures in Difmap. During this iterating process, the solution interval of an amplitude self-calibration was shortened from intervals as long as the whole observational time down to 15 minutes. Also, we conducted the two dimensional Gaussian model-fitting of the self-calibrated visibility data in the $(u, v)$-plane using the Difmap task modelfit.

\section{Results}

\section{Session A}

The position of the innermost component named hereafter JVN C1, which is located at $~$ 


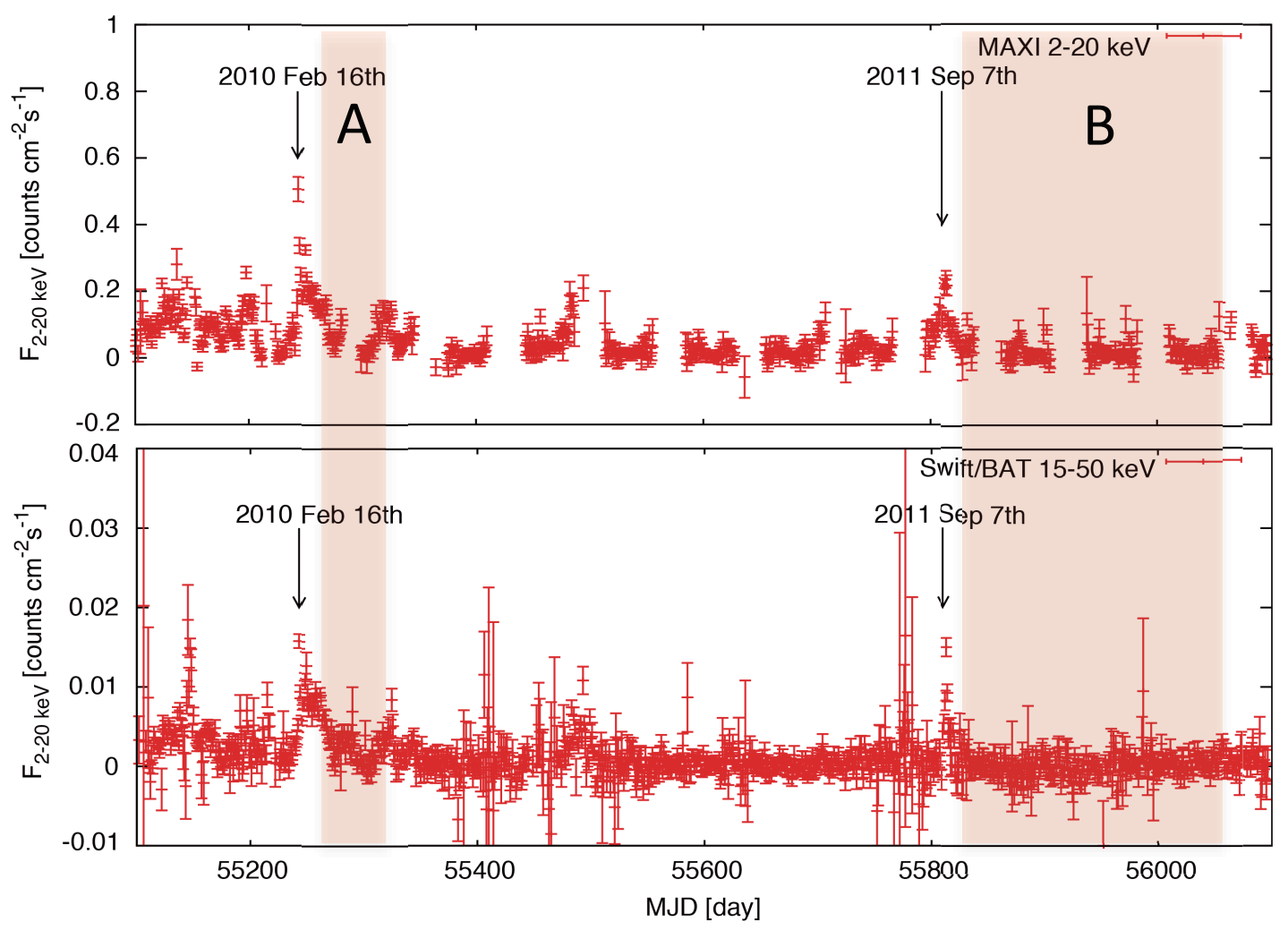

Figure 1: X-ray light curve of Mrk 421. Upper panel indicates the MAXI light curve (2-20 keV), and lower panel indicates the Swift/BAT light curve (15-50 keV: Swift/BAT transient monitor results provided by the Swift/BAT team). The shaded area show the period of our VLBI follow-up observations triggered by 2010 Feb flare (session A) and 2011 Sep flare (session B), respectively.

1.2 mas from the core, is plotted as a function of time in Figure 2 of Niinuma et al. [D]. As a result of the least-squares fit of the displacements of the position of JVN C1 relative to the core during 66 days (from March 7 to May 12 2010) under the assumption of linear motion of JVN C1, we obtained a proper motion of $\mu_{a}=-1.66 \pm 0.46 \mathrm{mas} \mathrm{yr}^{-1}$, which corresponds to an apparent velocity of $v_{a}=-3.48 \pm 0.97 c$ at the distance of Mrk 421. This negative velocity implies apparent inward motion of JVN C1.

\section{Session B}

The innermost component (named hereafter VERA C1, but which is the same component as JVN C1 obtained by the JVN observation in epoch A) shows large positional fluctuations of the angular distance from the core and the position angle relative to the core until 2012 February in Figure $\square$ on the left-hand side (shaded period). Also, a typical model-fitted image of Mrk 421 obtained by VERA is shown in Figure $\square$ on the right-hand side. We used two circular gaussian components to model-fit our data ([ए]6]). An estimation of the error for both the angular distance and the position angle, and the astrometric analysis has not been finished yet. 

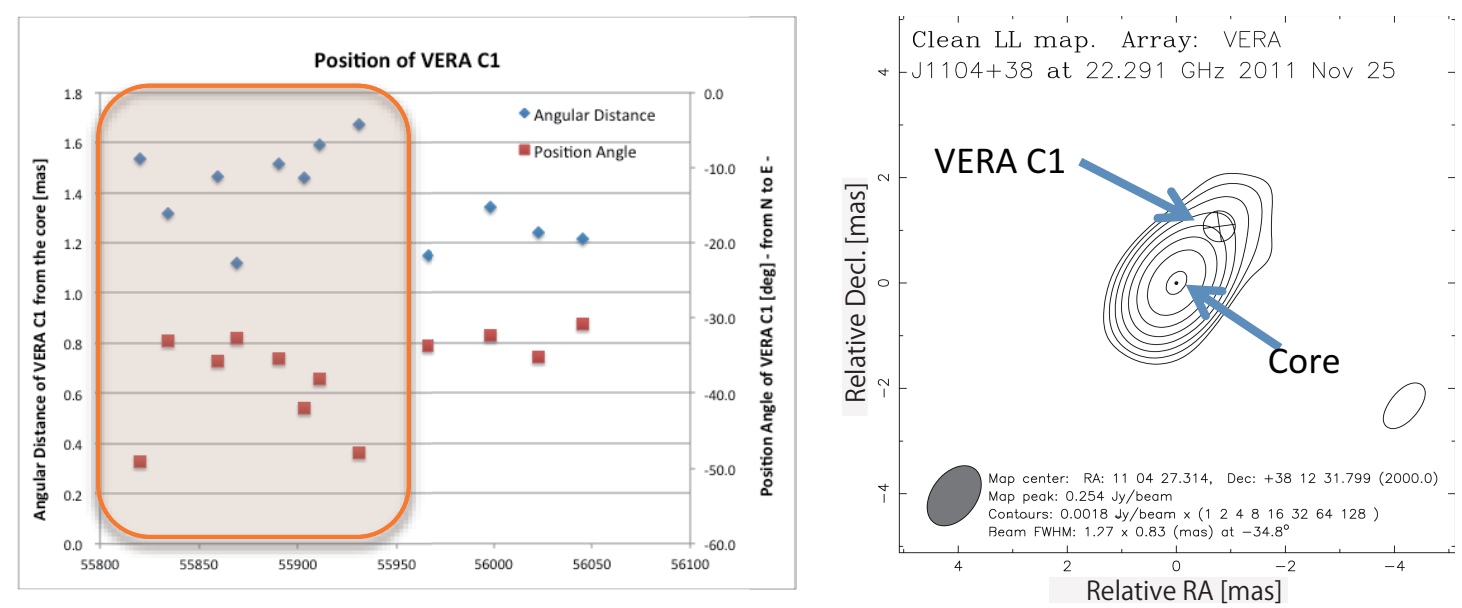

Figure 2: Left : the angular distance from the core (blue squares) and the position angle relative to the core (red diamonds) of VERA C1. The largest gap of the position angle can be seen on 2012 February 9. Also, the fluctuation of the angular distance between the X-ray flare (occurred on MJD 55811) and MJD 55950 seems to be larger than for the last four epochs [ए]]. Right : model fitted image of Mrk 421 obtained by our observations on 2012 February 9.

\section{Discussion \& conclusion}

An inward motion of the innermost component was detected in our VLBI images of the quick follow-up session A. As one of the possibilities, the inward motion implies that the superluminal knot associated with the largest X-ray flare was ejected in the core region of Mrk 421. In this case, the possibility that new component which shifted the centroid of the core faded within 0.2 years is considerable (see $[\square]$ ). Also, there is a possibility that the large positional fluctuation of the inner most component in session B shows the structural change occurring in the core region right after the large flare. We carried out multi-epoch VLBI observation for the high-energy flare of Mrk 421 at the shortest interval among the similar previous studies of Mrk 421. Quick follow-up observations and densely monitoring using VLBI for the 'large flares' of the nearby blazars are essential, since they may show new astrophysical behaviors of the blazars.

\section{Acknowledgements}

This research has made use of the MAXI data provided by RIKEN, JAXA and the MAXI team. This work was partially supported by Grant-in-Aid for Scientific Researchers (24540240, MK) from Japan Society for the Promotion of Science (JSPS). Swift/BAT transient monitor results are provided by the Swift/BAT team.

\section{References}

[1] Punch, M., et al., 1992, Nature 358, 477

[2] Gaidos, J. A., et al., 1996, Nature 383, 319

[3] Krennrich, F., et al., 2001, ApJ 575, L9 
[4] BŁażejowski, M., et al., 2005, ApJ 630, 130

[5] Lichti, G. G., et al., 2008, A \& A 486, 721

[6] Donnarumma, I., et al., 2009, ApJ 691, L13

[7] Niinuma K., et al., 2012, ApJ 759, 84

[8] Matsuoka, M., et al., 2009, PASJ 61, 999

[9] Swift/BAT database: http://heasarc.gsfc.nasa.gov/docs/swift/results/transients/

[10] Isobe N., et al. 2010, The Astronomer's Telegram, 2444, 1

[11] Hiroi K., et al. 2011, The Astronomer's Telegram, 3637, 1

[12] Isobe N., et al., 2010, PASJ 62, L55

[13] Doi A., et al. 2006, Proceedings of the 8th European VLBI Network Symposium,

[14] Kobayashi H., et al. 2003, New technologies in VLBI, 306, 367

[15] Nagai H., et al., 2010, PASJ 62, L11

[16] Niinuma K., et al., in prep. 